\title{
Assessment of the frequency of Staphylococcus aureus carriers and its antibiotic susceptibility in paramedical students in Rafsanjan University of Medical Sciences, Iran
}

\author{
Mohseni Moghadam F, MD ${ }^{1}$, Tashakori M, $\mathrm{PhD}^{2}$, Shahidi Zandi B, $\mathrm{MD}^{3}$, Hadavi M, MSc ${ }^{4^{*}}$, \\ Ranjbar E, BSc ${ }^{5}$, Shahidi Zandi Sh, Medical Student ${ }^{6}$ \\ 1-Facully Member, Dept. of Basic science, Paramedical Faculty, Rafsanjan University of Medical Sciences, Rafsanjan , Iran. \\ 2- Assistant Prof., Dept. of Basic Science, Paramedical Faculty ,Rafsanjan University of Medical Sciences, Rafsanjan, Iran. \\ 3 - MD in Medical Laboratory Basic Science, Rafsanjan University of Medical Sciences, Rafsanjan, Iran. 4- PhD Student, \\ Dept. of Anesthesiology, Paramedical Faculty, Rafsanjan University of Medical Sciences, Rafsanjan, Iran. 5- Student of \\ Paramedical, Rafsanjan University of Medical Sciences, Rafsanjan, Iran. 6- Medicine Student, Kerman University of \\ Medical Sciences, Kerman, Iran.
}

Received: October 2016, Accepted: December 2016

Background: Methicillin-resistant Staphylococcus aureus (MRSA) is resistant to most antibiotics and is an important pathogen of nosocomial infections. The prevalence of community (CA-MRSA) and hospital acquired methicillin-resistant $S$. aureus (HA-MRSA) infection is increased. In this study we investigated the frequency of MRSA colonization and its antibiotic susceptibility in students of Rafsanjan University of Medical Sciences, Iran.

Materials and Methods: In this cross-sectional study, we evaluated 200 nursing, midwifery and paramedical students. Nasal swabs were taken from all cases and were cultured on a blood medium agar. Methicillin resistance was confirmed using Oxacillin and cefoxcitin disks. Inducible clinadamycin resistance was identified using D-zone test. Demographic and specific information were collected by questionnaire. Data were analyzed by chi-square test.

Results: Among 200 studied cases, the frequency of nasal carriers for S. aureus was 5\%. Six (60\%) out of $10 \mathrm{~S}$. aureus isolates were MRSA strains. Fifty percent of MRSA and 25\% of methicillinsusceptible S. aureus (MSSA) were resistant to clindamycin. Four out of 6 strains of MRSA and 1 of the MSSA strains were resistant to erythromycin and D test was positive in $50 \%$ of cases.

Conclusions: Nasal carriers of the resistant strains of $S$. aureus are always a serious threat to themselves and others. The rate of MRSA colonization, especially clindamycin-resistant strains, was high among studied cases, emphesizing the need for screening S. aureus.
\end{abstract}

Keywords: Staphylococcus aureus, Antibiotic, Students

\section{Introduction}

In the Staphylococcus genus, Staphylococcus aureus species are known as the most important and the most common causes of hospital infections and infections acquired in the community around the world $(1,2)$. This microorganism is a non-motile, gram-positive cocci that can grow in different environmental conditions without spores. The major ecological habitat of S. aureus is in the anterior region of the nose and about $20 \%$ of the population are carriers. The bacteria causes a wide range of infections such as bacteremia, septicemia, infection of the skin, soft tissues, bone, and pneumonia and it can be transmitted through direct contact or through objects (3-5). In 1961, the first strains of methicillinresistant $S$. aureus (MRSA) were identified in Europe (6). This strain has spread widely since the 1980s and is resistant to all beta-lactams such as penicillins, cephalosporins, carbapenems and imipenems. Due to the

\footnotetext{
* Corresponding author: Maryam Hadavi, Dept. of Anesthesiology, Paramedical Faculty, Rafsanjan University of Medical Sciences, Rafsanjan, Iran.

E-mail: hadavimaryam@yahoo.com
} 
emergence of antibiotic-resistant strains of $S$. aureus, the number of effective antibiotics is declining in its treatment (7). The colonization rate of this organism is higher in medical staff so that one of the main sources of $S$. aureus infections is hospital personnel. These individuals are an important source for the spread of infections with hospital-acquired MRSA (HA-MRSA) in the patients (8). Community-acquired MRSA, (CA-MRSA) is not associated with risk factors such as hospitalization, dialysis, drug addiction, AIDS, and chronic diseases (9), but is a serious problem in closed communities like kindergartens, barracks, classrooms, poor urban communities, prisoners, and the centers where people are in close contact with each other $(10,11)$. Colonization of methicillinresistant strains in the community is a way to spread the bacteria in closed communities and infecting people. According to reports, the consequences of infection with MRSA are more severe than infection with MSSA, and can lead to bacteremia or endocarditis. Complications such as renal failure is higher among patients infected with MRSA compared to MSSA. Even mortality is significantly higher among patients with MRSA compared to MSSA (4). This study aimed to evaluate the nasal colonization of $S$. aureus and to investigate patterns of methicillin susceptibility among nursing, midwifery, and paramedical students.

\section{Materials and Methods}

This was a cross-sectional study, conducted on 200 students of Rafsanjan University of Medical Sciences, Iran, after receiving the license from the Ethics Committee of the University in 2015. The gender ratio and the number of students in each field were enrolled through stratified random sampling. The students who were hospitalized during the last month, received antibiotics in the past two weeks, and those students whose family members were hospital staff were excluded. The demographic characteristics including gender, field of study, and education level were obtained by a researcher-designed questionnaire.

After explaining the objectives of the study by the microbiology expert and obtaining consent from students, the anterior nares were sampled by a sterile swab moistened with nutrient broth. The samples were transferred to the laboratory in the transport medium. All samples were cultured in blood agar solidified medium. Colonies' growth was evaluated after 24 hours of incubation at $37{ }^{\circ} \mathrm{C}$.. They were also evaluated in terms of Gram stain and catalase test. DNase positive colonies, positive catalase, positive mannitol and coagulase were confirmed as $S$. aureus (12). In the next stage, a suspension of 0.5 MacFarld was prepared from all positive samples and they were transferred to Mueller-Hinton agar with $4 \%$ sodium chloride. Then, they were incubated using oxacillin disk (Mast England Company) for 24 hours. S. aureus was reported in case of growing in the medium containing oxacillin resistant to methicillin (13). Drug resistance of all $S$. aureus strains to vancomycin, gentamicin, clindamycin, ciprofloxacin, tetracycline, rifampin, teicoplanin, cotrimoxazol, erythromycin, and doxycycline was determined by disk diffusion method (Padtan Teb Company). Drug resistance and sensitivity were reported using a standard table in the form of resistant, susceptible, and intermediate (11).

D-test method was used to evaluate the inducible resistance to clindamycin. Bacteria were inoculatedand erythromycin and clindamycin discs were placed above them 20$15 \mathrm{~mm}$ apart. After incubation at $37{ }^{\circ} \mathrm{C}$ for 24 hours and examining the non-growth haloes and flatting the inhibitory disk adjacent to erythromycin disc, inhibition zone in the form of $\mathrm{D}$ was considered as a positive result (13).

Demographic data and test results were analyzed by SPSS, version 21 (IBM Corporation, USA). Chi-square test was used for categorical variables and $\mathrm{P}<0.05$ was considered significant. 


\section{Results}

From 200 studied students, 56 patients (28\%) were male and $144(72 \%)$ were female. Ten students $(5 \%)$ were colonized with $S$. aureus in the nose of which $2(3.6 \%)$ were men and 8 $(5.6 \%)$ were women. There was no significant association between gender and colonization with $S$. aureus $(\mathrm{P}<0.05)$ (Table 1). The colonization rate in students who had entered the hospital were $5.8 \%$, although among student who had not entered the hospital were lower $(4.2 \%)$. No significant difference was shown among studied cases $(\mathrm{P}<0.05)$.

Table 1: Frequency of methicillin-resistant $S$. aureus in students based on demographic characteristics

\begin{tabular}{|c|c|c|c|c|}
\hline \multicolumn{2}{|c|}{ Variable } & $\begin{array}{c}\text { S. aureus } \\
\mathrm{N}(\%)\end{array}$ & $\begin{array}{l}\text { MRSA } \\
\text { N (\%) }\end{array}$ & $\begin{array}{l}\text { MSSA } \\
\text { N (\%) }\end{array}$ \\
\hline \multirow{2}{*}{ Gender } & Female & $8(6.5)$ & $5(62.5)$ & $3(37.5)$ \\
\hline & Male & $2(3.5)$ & $1(50)$ & $1(50)$ \\
\hline \multirow{2}{*}{ Admission to hospital } & Yes & $6(5.8)$ & $5(62.5)$ & $3(37.5)$ \\
\hline & No & $4(4.2)$ & $1(50)$ & $1(50)$ \\
\hline \multirow{6}{*}{ Field of study } & Nursing & $2(5.4)$ & $2(100)$ & $0(0)$ \\
\hline & Midwifery & $1(4.5)$ & $1(100)$ & $0(0)$ \\
\hline & Anesthesiology & $2(10.5)$ & $1(100)$ & $1(50)$ \\
\hline & Surgical technologist & $1(2.2)$ & $1(100)$ & $0(0)$ \\
\hline & Radiology & $2(4.3)$ & $0(0)$ & $2(100)$ \\
\hline & Laboratory sciences & $2(4.4)$ & $1(50)$ & $1(50)$ \\
\hline Total & & $10(5)$ & $6(60)$ & $4(40)$ \\
\hline
\end{tabular}

MRSA: Methicillin-resistant S. aureus, MSSA: Methicillin-sensitive S. aureus

With regard to field of study, 2 (4.4\%) from 45 laboratory sciences students, $2(10.5 \%)$ from 19 anesthesiology students, 1 (3.2\%) from surgery room students, 2 (4.5\%) from 37 nursing students, 2 (4.3\%) from radiology students, and 1 (4.5\%) from 22 midwifery students were carriers of $S$. aureus. There was no significant association between the educational field and the nasal colonization with $S$. aureus ( $<0.05)$. MRSA was isolated in $6(60 \%)$ and MSSA was isolated in $4(40 \%)$ of the carriers (Table 2).

Table 2: Sensitivity of antibiotic in methicillin-resistant $S$. aureus acquired from community

\begin{tabular}{|c|c|c|c|c|c|}
\hline \multirow{3}{*}{ Antibiotics } & \multicolumn{3}{|c|}{ S. aureus $(\mathrm{N}=10)$} & \multicolumn{2}{|c|}{$\operatorname{MRSA}(N=6)$} \\
\hline & $\mathbf{S}$ & I & $\mathbf{R}$ & $\mathbf{S}$ & $\mathbf{R}$ \\
\hline & $\mathbf{N}(\%)$ & $\mathbf{N}(\%)$ & $\mathrm{N}(\%)$ & $\mathbf{N}$ & $\mathbf{N}$ \\
\hline Oxacillin ( OX) & $3(30)$ & $1(10)$ & $6(60)$ & 0 & 6 \\
\hline $\begin{array}{c}\text { Clindamycin } \\
(\mathbf{C C})\end{array}$ & $7(70)$ & $3(30)$ & $0(0)$ & 6 & 0 \\
\hline $\begin{array}{c}\text { Erythromycin } \\
\text { (E) }\end{array}$ & $3(30)$ & $3(30)$ & $4(40)$ & 3 & 3 \\
\hline $\begin{array}{c}\text { Ciprofloxacin } \\
\text { (CP) }\end{array}$ & $8(80)$ & $1(10)$ & $1(10)$ & 6 & 0 \\
\hline $\begin{array}{c}\text { Cotrimoxazole } \\
(\text { SXT) }\end{array}$ & $2(20)$ & $5(50)$ & $3(30)$ & 5 & 1 \\
\hline Vancomycin (V) & $10(100)$ & $0(0)$ & 0 & 6 & 0 \\
\hline Rifampin (RA) & $10(100)$ & 0 & 0 & 6 & 0 \\
\hline $\begin{array}{c}\text { Gentamicin } \\
(\text { GM) }\end{array}$ & $8(80)$ & $1(10)$ & $1(10)$ & 5 & 1 \\
\hline Tetracycline (TE) & $5(50)$ & $2(20)$ & $3(30)$ & 4 & 2 \\
\hline Ticoplanin (TEC) & $10(100)$ & 0 & 0 & 6 & 0 \\
\hline
\end{tabular}


Nasal colonization with MRSA was $2.8 \%$ in female carriers and $1.8 \%$ in male carriers. Among the students who had entered the hospital, 2.9\% were MRSA carriers while this rate was $2.1 \%$ in students who had not entered the hospital, which was not statistically significant $(\mathrm{P}<0.05)$.

Clindamycin resistance was observed in $50 \%$ of MRSA and in 25\% of MSSA isolates. Four of 6 MRSA and 1of 4 MSSA isolates were resistant to erythromycin and D-test was positive in $50 \%$ of the cases (Table 2).

\section{Discussion}

$S$. aureus is one of the most important pathogens in the community and the increasing incidence of resistant strains to antibiotics, particularly methicillin, is the most urgent health problem in the world. MRSA are studied in two groups of HA-MRSA and CAMRSA (4). Studies have shown that CAMRSA has the capability to disseminate to the hospital and cause nosocomial infections (14). This study was conducted to determine the frequency of $S$. aureus nasal carriers among nursing, midwifery, and paramedical students and to compare the resistance of isolated $S$. aureus strains to different antibiotics, especially methicillin, in different groups of students. In this study, 5\% of nursing, midwifery, and paramedical (including laboratory sciences, anesthesia, surgery room, radiology) students were carriers of $S$. aureus. There is no similar study on nursing, midwifery, paramedical students, but the prevalence of nasal carriage in this study was lower than the previous studies on medical students and mostly healthcare workers across the country. The prevalence of nasal carriage in various medical schools in Hamedan was $25.2 \%$ (15). This value was $31.7 \%$ among health care workers in Hamedan, and $31 \%$ in Tehran (16). However, Zia sheikh aleslami et al. reported that the nasal carriage was $20 \%$ among healthcare workers in Rafsanjan , which was lower than other cities (17).
The results of studies conducted in other countries have reported higher prevalence compared to the results of this study. In China, S. aureus nasal carriage was reported $43 \%$ among students (18), while in Brazil, it was reported to be $40.8 \%(18,19)$. S. aureus nasal carriage in an area depends on factors such as the socioeconomic status of residents, paying attention to health, and antibiotics decomposition, and this could explain the lower prevalence of nasal carriage among students and health care workers in some areas compared to the others.

The prevalence of nasal carriage among students who had already entered hospital was not significantly different compared to those who had not entered hospital. In Ghasemian study, there was no significant relationship between age, gender, and work experience with nasal carriage (20). In addition, Hashemi showed that there was no significant association between the incidence of bacteria carriers and the employment duration in hospital personnel (15).

In this study, $60 \%$ of $S$. aureus isolates were methicillin resistant of which, $2.9 \%$ was in students who had not entered the hospital (new entrants) and $2.1 \%$ was in students who had entered the hospital and there was no statistically significant difference between these two groups.

The prevalence of MRSA in this study was much more than expected, and it was nearly similar to the prevalence in hospital personnel in studies conducted in Ahwaz and Rafsanjan $(17,21)$. In another study in Iran, clinically isolated MRSA was $88 \%$. It has been reported that the prevalence of MRSA is increasing in Iran (22). In our study, there was no significant relationship between gender and prevalence of MRSA. Other studies have also reported a similar result (23-25). In this study, resistance to vancomycin was not observed, which was similar to previous studies in Iran $(23,26)$, and another study conducted in eight Asian countries and in India . This is probably due to reduced inappropriate use of antibiotics or raising public awareness on the appropriate 
use of antibiotics $(27,28)$. In this study, all isolated $S$. aureus strains were sensitive to rifampin and teicoplanin. However, other studies have not observed similar resistance pattern to these two drugs (26).

In this study, the induced resistance to clindamycin in MRSA was 60\%. Some authors have reported increasing resistance to clindamycin in CA-MRSA (8).

\section{Conclusion}

Since the prevalence of infection with MRSA in community acquired infections is high, it is important to correctly identify the antibiotic resistance of isolated $S$. aureus from patients to ensure the proper treatment for infections caused by this organism. Further studies are warranted to establish a better judgment about the prevalence of MRSA in the society.

\section{Acknowledgments}

We would like to acknowledge the Rafsanjan University of Medical Sciences for sponsoring this study. Also, we would like to thank Mrs. Somayeh Sakhvidi, the microbiological expert, for sampling and testing.

Conflict of interest: None declared.

\section{References}

1. Bennett JE, Dolin R, Blaser MJ. Principles and practice of infectious diseases. $7^{\text {th }}$ ed. Elsevier Health Sciences; 2014. (2-Volume Set).

2. Kasper DL, Fauci AS, Hauser SL, Longo DL, Jameson JL, Loscalzo J. Harrison's manual of medicine. $19^{\text {th }}$ ed. New York City, United States: McGraw Hill Education; 2015.

3. Brooks GF, Carroll KC, Butel J, Morse S. Jawetz, Melnick and Adelberg's medical microbiology. $24^{\text {th }}$ ed. New York City, United States: McGraw Hill Education, 2007.

4. Diederen BMW, Kluytmans JAJW. The emergence of infections with communityassociated methicillin resistant Staphylococcus aureus. Journal of Infection 2006; 52(3):15768 .

5. Kasper D, Fauci A, Hauser S, Longo D, Jameson JL, Loscalzo J. Harrison's principles of internal medicine. $19^{\text {th }}$ ed. New York City, United States: McGraw Hill Education; 2015.

6. Patricia Jevons M. "Celbenin"-resistant Staphylococci. Br Med J 1961; 1(5219):124-5.

7. Wunderink RG. How important is methicillinresistant Staphylococcus aureus as a cause of community-acquired pneumonia and what is best antimicrobial therapy? Infect Dis Clin North Am 2013; 27(1):177-88.

8. Crum NF, Lee RU, Thornton SA, Stine OC, Wallace MR, Barrozo C, et al. Fifteen-year study of the changing epidemiology of methicillin-resistant Staphylococcus aureus. Am J Med 2006;119(11):943-51.

9. Salgado CD, Farr BM, Calfee DP. Community-acquired methicillin-resistant Staphylococcus aureus: a meta-analysis of prevalence and risk factors. Clin Infect Dis 2003; 36(2):131-9.

10. Scheurich D, Woeltje K. Skin and soft tissue infections due to CA-MRSA. Mo Med 2008; 106(4):274-6.

11. Turnidge JD, Bell JM. Methicillin-resistant Staphylococcal aureus evolution in Australia over 35 years. Microb Drug Resist 2000; 6(3):223-9.

12. Tille P. Bailey \& Scott's diagnostic microbiology. $13^{\text {th }}$ ed. United States: Elsevier Health Sciences; 2013.

13. Clinical and Laboratory Standards Institute. M100-S24: Performance standards for antimicrobial susceptibility testing, twentyfourth informational supplement. $24^{\text {th }}$ ed. 950 West Valley Road, Suite 2500, Wayne Pennsylvania 19087 USA: nat'l comm clinical lab standards; 2014.

14. Carleton HA, Diep BA, Charlebois ED, Sensabaugh GF, Perdreau-Remington F. Community-adapted methicillin-resistant Staphylococcus aureus (MRSA): population dynamics of an expanding community reservoir of MRSA. J Infect Dis 2004; 190(10):1730-8.

15. Hashemi SH, Seifrabiei MA, Ahmadi S, Alikhani MY. Frequency of nasal carriage of Staphylococcus aureus and its antimicrobial resistance in Hamadan's medical students. Scientific Journal of Hamadan University of Medical Sciences 2012; 19(3):36-40.

16. Rahbar M, Yaghoobi M, Kia-Darbandsari B. Prevalence of nasal carriage of Staphylococcus aureus and susceptibility of isolates to methicillin and mupirocin among healthcare workers in an Iranian Hospital. Infect Control Hosp Epidemiol 2006; 27(3):323-5.

17. Ziasheykh Aleslami N, Rezaeian M, Tashakori M. Determination of the prevalence of Staphylococcus aureus nasal carriers and 
antibiotic resistance pattern in clinical wards staff of Ali-Ebne Abitaleb Hospital, Rafsanjan. Journal of Rafsanjan University of Medical Sciences 2009; 8(1):27-36.

18. Ma XX, Sun DD, Wang S, Wang ML, Li M, Shang $\mathrm{H}$, et al. Nasal carriage of methicillinresistant Staphylococcus aureus among preclinical medical students: epidemiologic and molecular characteristics of methicillinresistant S. aureus clones. Diagn Microbiol Infect Dis 2011;70(1):22-30.

19. Prates KA, Torres AM, Garcia LB, Yamada Ogatta SF, Cardoso CL, Bronharo Tognim MC. Nasal carriage of methicillin-resistant Staphylococcus aureus in university students. Braz J Infect Dis 2010; 14(3):316-8.

20. Ghasemian R, Najafi N, Makhlough A, Khademloo M. Frequency of nasal carriage of Staphylococcus aureus and its antimicrobial resistance pattern in patients on hemodialysis. Iran J Kidney Dis 2010; 4(3):218-22.

21. Alavi SM, Rajabzadeh AR, Dezfoulian A, Haghighizadeh MH. Determination of nasal carriage of Staphylococcus aureus and antimicrobial resistance among hospital personnel in razi hospital Ahwaz, spring 2003. Jundishapur Scientific Medical Journal 2006; 5(1):378-84.

22. Saderi H, Owlia P, Jalali Nadoushan MR. Difference in epidemiology and antibiotic susceptibility of methicillin resistant and methicillin susceptible Staphylococcus aureus isolates. Arch Clin Infect Dis 2009; 4(4):21923.

23. Fatholahzadeh B, Emaneini M, Gilbert G, Udo E, Aligholi M, Modarressi MH, et al. Staphylococcal cassette chromosome mec
(SCC mec) analysis and antimicrobial susceptibility patterns of methicillin-resistant Staphylococcus aureus (MRSA) isolates in Tehran, Iran. Microb Drug Resist 2008;14(3):217-20.

24. Graffunder EM, Venezia RA. Risk factors associated with nosocomial methicillinresistant Staphylococcus aureus (MRSA) infection including previous use of antimicrobials. J Antimicrob Chemother 2002; 49(6):999-1005.

25. Mohraz M, Jonaidi N, Rasoulinejad M, Broum MA, Aligholi M, Shahsavan Sh. Determination of prevalence of methicillin resistant staphylococcus infections through measurement of mics of $\mathrm{s}$. aureus isolates imam hospital (november 2001 to january 2003). Tehran University Medical Journal 2003; 61(3):182-92.

26. Tashakori M, Mohseni Moghadam F, Ziasheikholeslami N, Jafarpour P, Behsoun M, Hadavi M, et al. Staphylococcus aureus nasal carriage and patterns of antibiotic resistance in bacterial isolates from patients and staff in a dialysis center of southeast Iran. Iran J Microbiol 2014; 6(2):79-83.

27. Shagufta Naseer B, Jayaraj YM. Nasal carriage of methicillin resistant Staphylococcus aureus isolates from intensive care unit patients. Research Journal of Biological Sciences 2010; 5(2):150-4.

28. Song JH, Hsueh PR, Chung DR, Ko KS, Kang CI, Peck KR, et al. Spread of methicillinresistant Staphylococcus aureus between the community and the hospitals in Asian countries: an ANSORP study. J Antimicrob Chemother 2011; 66(5):1061-9. 